\title{
Hollow Cathode Arcs
}

\author{
A test-bench for plasma-wall interaction studies and new diagnostic equipment
}

\section{H. Kempkens and J. Uhlenbusch, Düsseldorf}

(Institute of Laser and Plasma Physics, University of Düsseldorf)

Magnetized hollow cathode arcs (HCA) were first used in thermonuclear experiments at the turn of the 1950s and later found a wide application because of their advantageous properties. These include good stability, high degree of excitation and ionisation, excellent access to the actual plasma column for diagnostic experiments and long burning time. They have served as sources of hot neutrals [1] and ions [2], as pulsed lamps [3], for isotope separation [4], in metal vapour lasers [5] and to study plasma-wall interaction processes.

In a typical plasma, the discharge pressure can be chosen to be within the range $1-10^{5} \mathrm{~Pa}$ and the electron density from $10^{18}-10^{21} \mathrm{~m}^{-3}$. The electron temperature reaches 5-10 eV, while heavy particle temperatures stay below $1 \mathrm{eV}$. Arc currents of $100 \mathrm{~A}$ are typical, the arc voltage is in the order of $10-100 \mathrm{~V}$ and magnetic fields from $10^{-2}-1 \mathrm{~T}$ are common. Electrons and ions reach a velocity of about $10^{4} \mathrm{~m} / \mathrm{s}$.

\section{HCA Geometry}

Hollow cathode arcs can be subdivided into longitudinal and transversal arrangements [6], but in the following, a longitudinal set-up is considered, Fig. 1. The plasma is generated within the hot hollow cathode (region I), and escapes into the actual discharge column (region II) which is terminated just by the anode or, depending on the radial plasma extension near the anode, by the anode fall (region III) as indicated in Fig. 1. The cold neutral gas enters the hollow cathode, becomes energised and partly ionised and leaves the cathode orifice at a high speed. Within the discharge column, a radial diffusion of charge carriers as well as neutrals occurs leading to radial plasma expansion and, as a consequence, a conical shape of the plasma (region II). The distance between the anode and the cathode as well as the anode diameter have a decisive influence on the plasma behaviour in the anodic region.

In HCA with a moderate electrode spacing, large anode diameter and relatively high magnetic field strength, the column plasma continuously recombines on the anode surface (the dashed plasma configuration in Fig. 1). With increase of the electrode spacing, a plasma distribution as shown with solid lines in Fig. 1 is observed. The plasma circulates around the anode and recombines on the surface of the insulator covering its sides. In front of the flat part of the anode in the region marked by the dashed-dotted line (region III), the electron density is reduced which strongly influences the ion motion and the radiative properties of the plasma.

\section{Inner Part of the Hollow Cathode}

The basic physics of plasma generation within region I is summarized in [6]. Numerical studies valid for different gases, pressures and discharge conditions were published by Baksht and Rybakov [7] and their results are briefly described here.
Fig. 1 - Scheme of a Hollow Cathode Arc discharge.

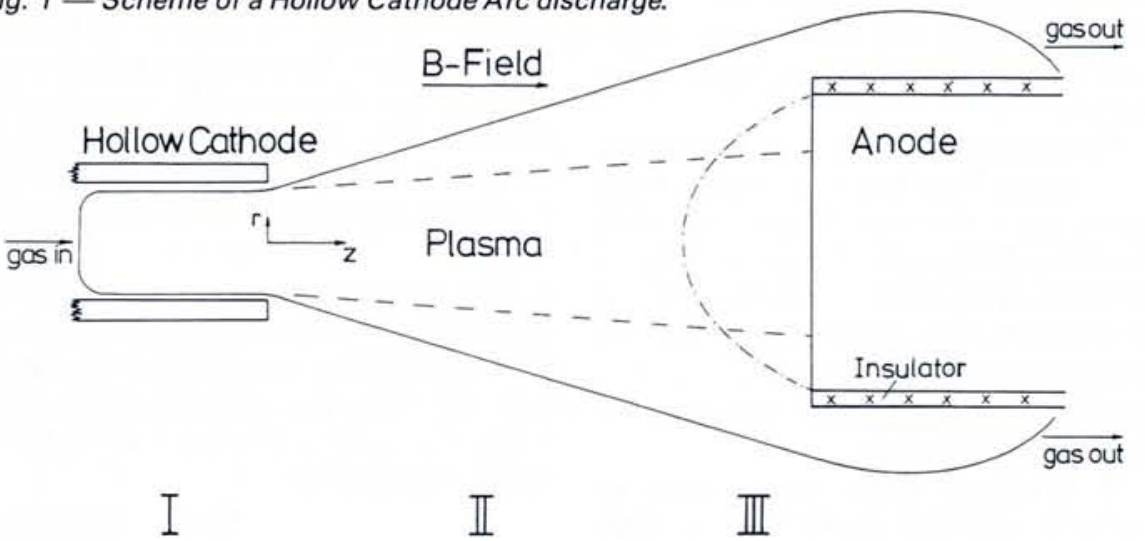

a

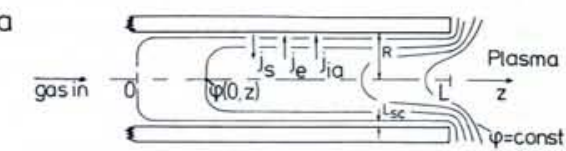

b

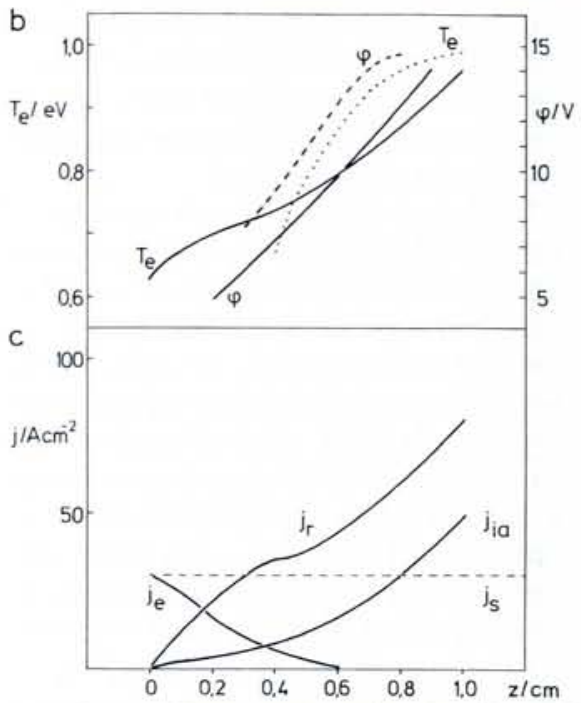

Fig. 2 - a) Cross-section showing lines of equal potential $\rho$,

$j$, total radial current density,

$j_{s}$ electron current density by thermal emission,

$j_{e}$ electron current density leaving the inner cathode plasma impinging on the inner cathode wall,

$j_{i a}$ ion current density leaving the inner cathode plasma impinging the inner cathode wall,

$R$ radius of the cathode orifice,

$L_{s c}$ space charge layer thickness.

b) Axial distribution of plasma potential $\rho$ and electron temperature $T_{e}$; calculated values (solid lines) compared with measured (broken lines) for $R=0.15 \mathrm{~cm}, T_{\text {Cathode }}$ $=0.1 \mathrm{eV}, p=250$ torr, and $j_{s}=30 \mathrm{~A} / \mathrm{cm}^{2}$ [7].

c) Axial distribution of the various current densities contributing to the total radial current density $j_{r}$.

The hollow cathode is assumed to be a narrow cylindrical cavity, see Fig. $2 a$, whose length $L$ is substantially greater than its radius $R$. For $R / L \ll 1$ a radial change in the potential occurs in a space charge layer of thickness $L_{S C}$, whilst throughout the range $0 \leq r \leq R-L_{\mathrm{SC}}$ a quasineutral plasma is assumed. In the plasma bulk, the potential, temperature of the particles, their densities and the current densities depend only on the 
axial coordinate $z$. Following this one dimensional approach, the conservation laws for the mass, charge, momentum and energy can be solved simultaneously to give the most important plasma parameters inside the hollow cathode. Here it is assumed that the cathode emission current density $j_{\mathrm{s}}$ is constant over the length of the cathode and independent of the operating conditions, see Fig. 2c. This is true for a short pulse operation mode. For CW operation, an additional energy balance of the hollow cathode tube leading to a $z$-dependent temperature profile of the cathode and, also the electron emission process must be solved.

It is worth noting that the total current leaving the hollow cathode orifice is not, as one could expect, proportional to $j_{\mathrm{s}}$ but is dominated rather by the ion current density $j_{\mathrm{ia}}$. The latter grows when approaching the exit region of the hollow cathode because of an increase in the electron density and temperature, see Fig. $2 b$. The cross-section averaged temperatures of ions $T_{\mathrm{i}}$ and neutrals $T_{n}$ stay below the electron temperature, in particular at pressures below $10^{3} \mathrm{~Pa}$. When approaching the $10^{5} \mathrm{~Pa}$ region and electron temperatures beyond, say, $10^{4} \mathrm{~K}$, the difference between $T_{\mathrm{e}}$ and $T_{\mathrm{i}}$, $T_{n}$ becomes marginal.

With respect to momentum transfer, the hollow cathode tube acts as a thruster which transforms the thermal energy into directed kinetic energy of the plasma leaving the hollow cathode orifice. The plasma velocity there reaches the critical value (ion acoustic velocity):

$$
V_{\mathrm{c}}=\left[\left(\gamma k_{\mathrm{B}} T_{\mathrm{i}}+k_{\mathrm{B}} T_{\mathrm{e}}\right) / M\right]^{1 / 2}
$$
where $\gamma$ is the adiabatic exponent and $M$ the atomic weight of the working gas.

\section{Column of the HCA}

Now we pay attention to region II, see Fig. 1. The accelerated and heated plasma leaving the cathode orifice expands into a large vessel under lowered pressure conditions, guided by an external magnetic field. In most cases the electrons are strongly influenced by the field i.e., the product of the Larmor frequency and average time between electron collisions is larger than unity. For the ions the product is much smaller than one and the weakly influenced ions suffer a strong radial drift after having left the hollow cathode.

Because the strongly influenced electrons remain near the discharge centre, a negative charge cloud develops there, while off-centre, positive charge clouds form. The resulting inward directed radial electric field retains the ions and

\section{ETH - ZÜRICH}

\section{Postdoctoral Position \\ in Particle (Intermediate Energy) Physics}

The Institute for Intermediate Energy Physics of the ETH Zürich has an opening for an experimental physicist at the postdoctoral level. The research is being conducted at the Paul Scherrer Institute (PSI, formerly SIN). The present project is an investigation of the pion-nucleon interaction from $X$-ray precision measurements of pionic hydrogen. $Q C D$ oriented questions are of central interest (chiral perturbation theory, $\sigma$ term, structure of the proton).

The candidate is expected to have research experience in particle or nuclear physics. For more information please contact

Prof. H.J. Leisi, Tel. (56) 993296.

Applications containing a curriculum vitae and names of referees should be sent to:

PSI

Würenlingen/Villigen

Personnel Division

CH-5232 Villigen PSI Switzerland

Code 28E

\section{University of Bergen}

\section{CHAIR IN EXPERIMENTAL PHYSICS; ELECTRO-OPTICS}

Department of Physics, University of Bergen, Norway invites applications for a vacant chair in experimental physics; electro-optics. Basic research interests with a technological applicability are particularly appropriate. We specifically seek candidates in solid state optical physics with emphasis on optical sensors.

Salary is on the scale B5, Nkr. 269642 p.a. gross, of which Nkr. 5104 p.a. are paid in pension contributions.

The chair will be appointed on the understanding that any changes in scientific duties, pension or retiring age made by law or by the King with the agreement of Parliament are to be accepted without compensation.

Applicants should submit five copies of scientific work - published or unpublished - which they wish to be considered for the appointment as well as six copies of a list of all scientific contributions with information on where they were published. Scientific contributions are to be submitted, in numbered order and in five groups, to the science faculty of the University of Bergen within one month of the closing date for applications. Scientific manuscripts in preparation may be submitted within three months of the closing date for applications provided notice of intent is given on submitting the other publications. Applicants are otherwise referred to the current rules for the procedure to be followed in the appointment of professorships and readerships. A resume of the vacant chair can be obtained on request from:

Sekretariatet for Det matematisk-naturvitenskapelige fakultet,

Postboks 25, - Universitetet, 5027 Bergen, Norway.

Should the evaluation committee find several applicants to be equally well qualified for the position, the University Senate (Kollegiet) will adhere to the regulations in the University of Bergen Equal Employment Opportunities agreement (Særavtale om likestilling).

Applications, which must include a complete curriculum vitae, should be addressed to the King and be sent together with relevant certificates and one copy of a list of publications to

Det matematisk-naturvitenskapelige fakultet,

Postboks 25, - Universitetet, 5027 Bergen, Norway,

by 2 May, 1989. 


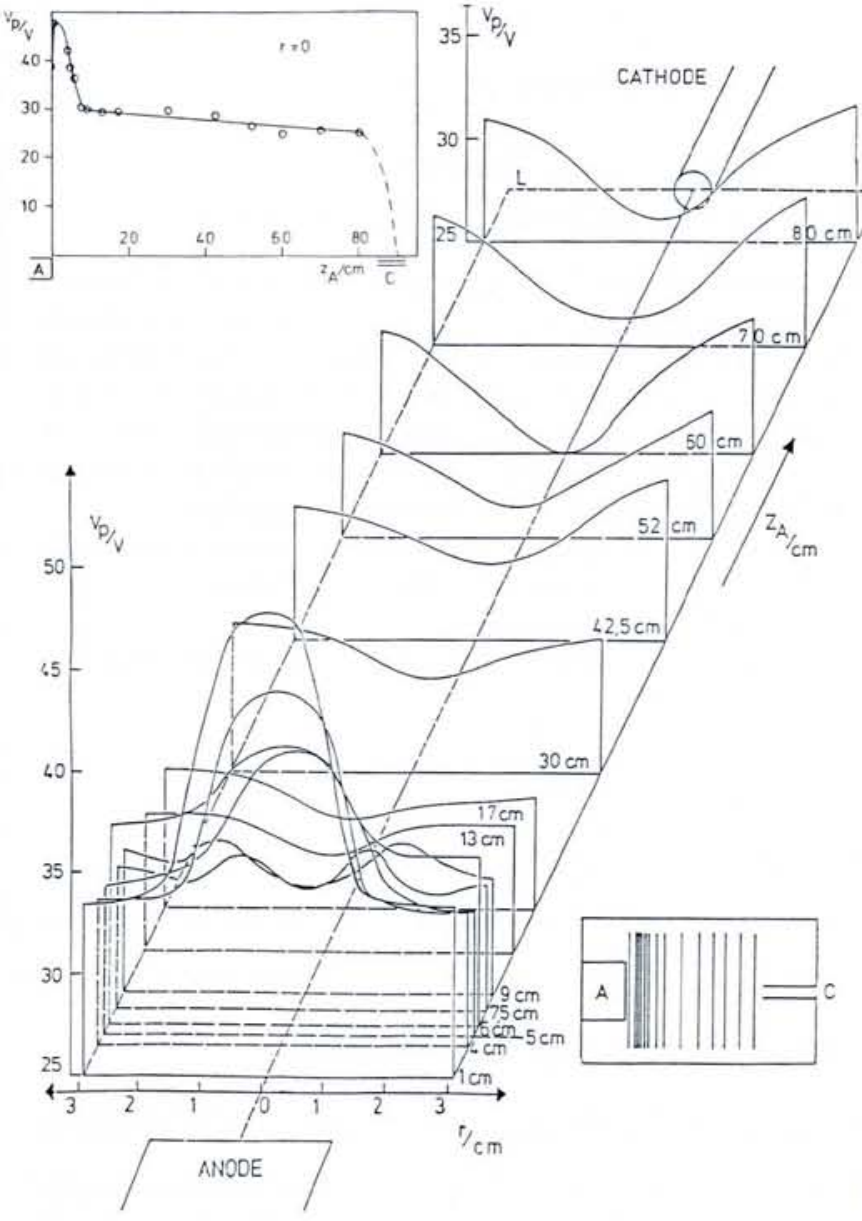

leads to an enhanced radial diffusion of electrons, see Fig. 3. Radial diffusion is the Bohm diffusion governed by a diffusion coefficient:

$$
D=k_{\mathrm{B}} T_{\mathrm{e}} / 16 e B
$$

The radial electric field cooperating with the axial B-field generates an azimuthal drift of electrons and ions leading to plasma rotation around the B-lines according to the right hand rule.

The radial diffusion of charge carriers results, to a fair degree, in a Gaussian spatial distribution of electrons and ions. The half-width of this distribution grows nearly exponentially with increasing distance from the cathode orifice. Because the charge carrier production and losses are negligible in region II (at least at low pressure) the electron density along the arc axis decreases rapidly when moving away from the cathode towards the anode.

The radial electronic temperature profile is nearly Gaussian, too, but the local balance of energy with Joule heating and radial as well as strong axial losses parallel to the B-field regulate the distribution in such a way that a uniform behaviour in the axial direction results.

All ions reaching the anode surface recombine and generate a cloud of neutrals which surrounds the anode. If the inter-electrode spacing is small, the area

\section{University of Zürich}

\section{Postdoctoral Position}

The physics institute expects to have a postdoctoral position available for a young, creative experimental physicist to do research on a 'Fifth Force' experiment. Please send applications to:

Prof. W. Kündig, Physics Institute, University of Zürich

Schönberggasse 9,

CH-8001 Zürich, Switzerland,

Tel. 01-257 2931.
Fig. 4-Measured spatial distribution of the electron den sity $n_{e}$ after [10], denotation and parameters see Fig. 3.
Fig. 3-Measured spatial distribution of the plasma potential $V_{p}$ after [8]; inter-electrode spacing $L=0.85$ $m$, arc current $I=60$ $A$, discharge pressure $p=1.338 \mathrm{~Pa}$, gas volume rate $Q=2.5$ $\mathrm{Nm}^{2} / \mathrm{s}$, magnetic field strength $B=0.074 T$.
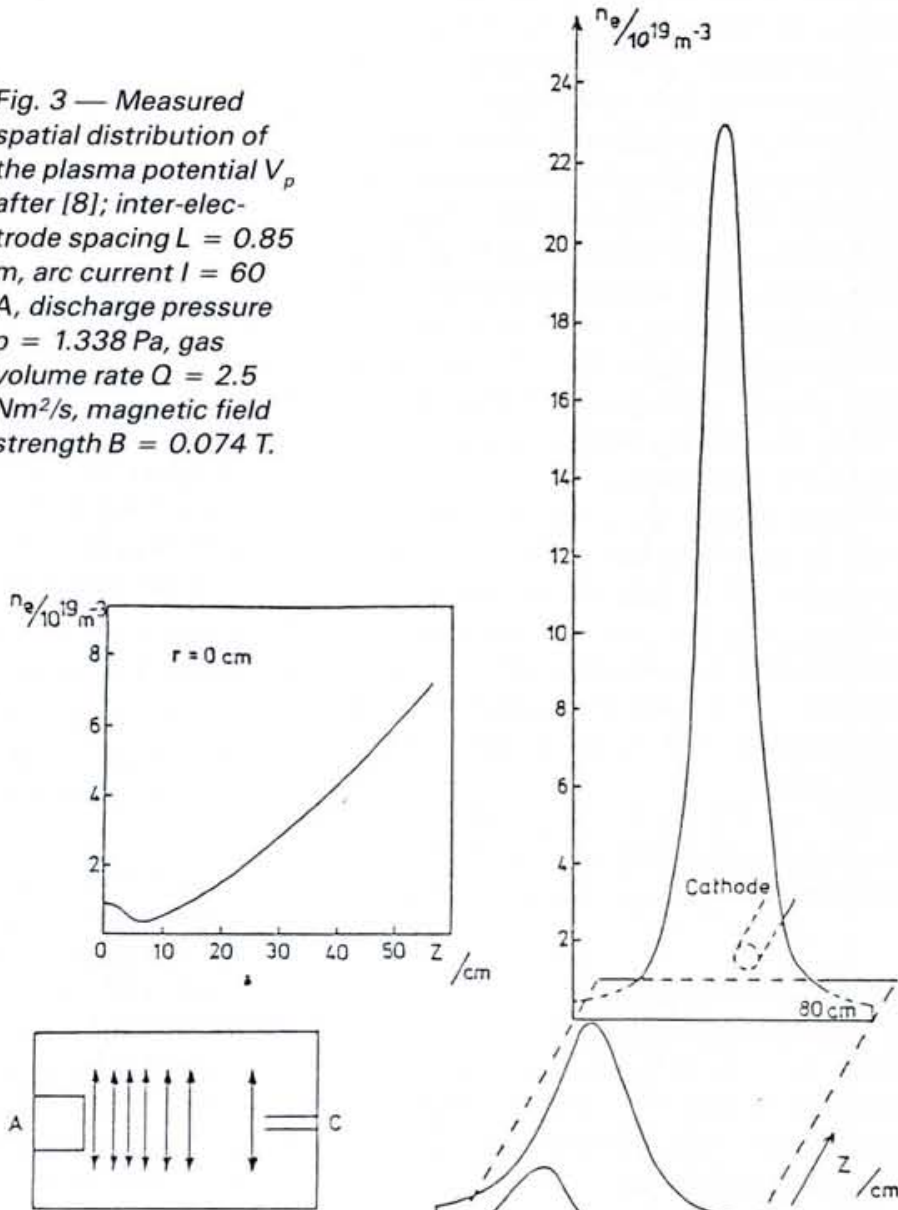

1 
of recombination zone is small too, and the electron densities in front of the anode are relatively high.

\section{Anodic Phenomena}

An entirely new situation is encountered if for large inter-electrode distances the area of the recombination zone becomes larger than the flat anode surface. Now the plasma envelops partially the anode and recombines on the insulated (quartz) side wall of the anode cylinder. This portion of the plasma cannot contribute to the arc current. The axial magnetic field inhibits diffusion of electrons moving backwards towards the cathode into the dashed-dotted region III, see Fig. 1, reducing the electron density there. By analogy to the mechanism occurring near a limiter of tokamak discharges, we call this phenomenon the scrape-off effect. Fig. 4 showing the measured electron density field gives evidence for these scraped-off electrons.

How does HCA manage to keep the arc current going in the scrape-off mode with strongly reduced electron density? The only way is to enhance the electron temperature in region III in order to push up the drift velocity of electrons, see Fig. 5. Such a temperature jump enlarges additionally the ionisation rate leading to an increase in the number of charge carriers near the anode, a fact which can be recognized by inspection of Fig. 3 .

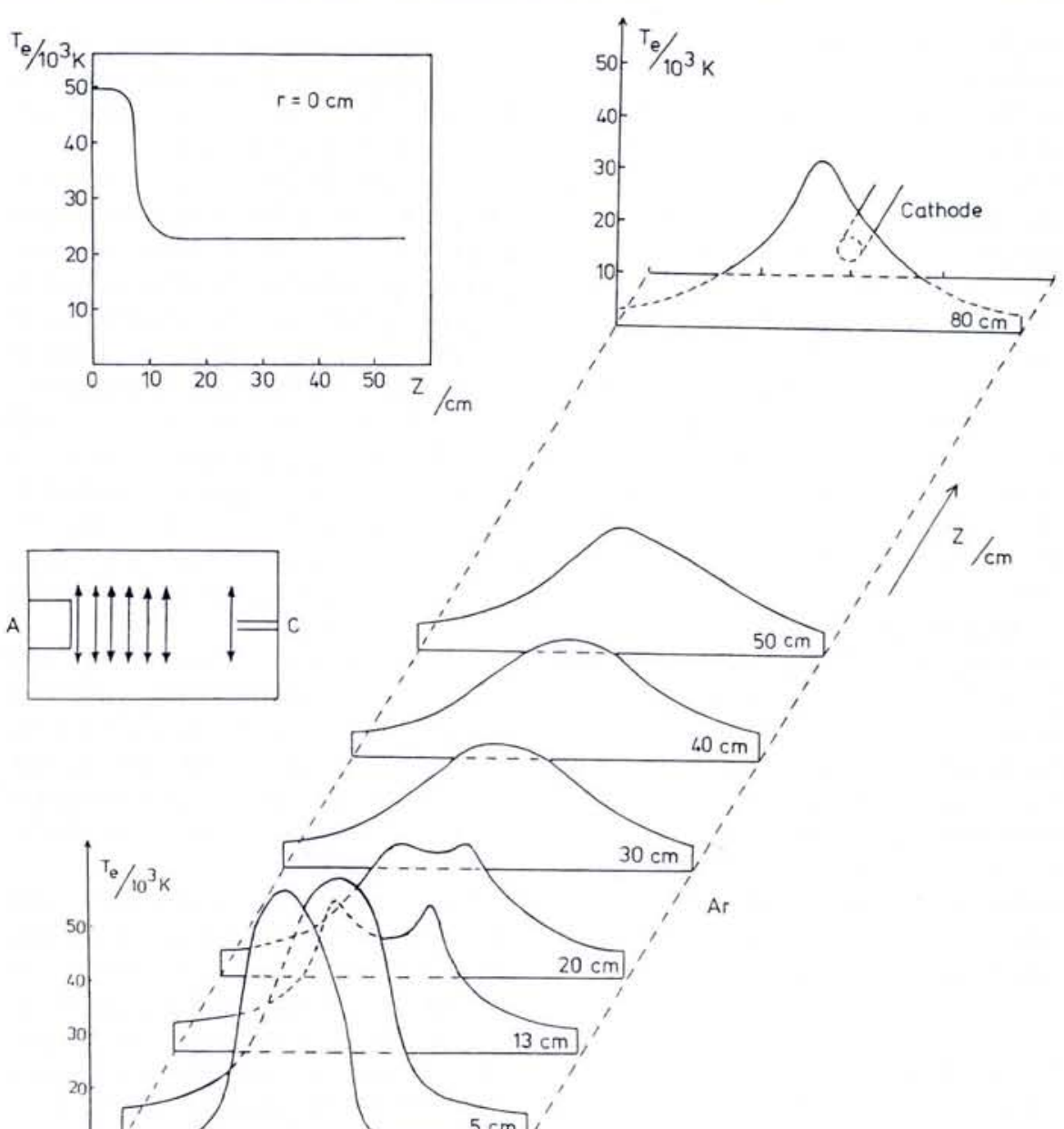

Fig. 5-Measured spatial distribution of the electron temperature $T_{e}$ after [10], denotation and parameters see Fig. 3.

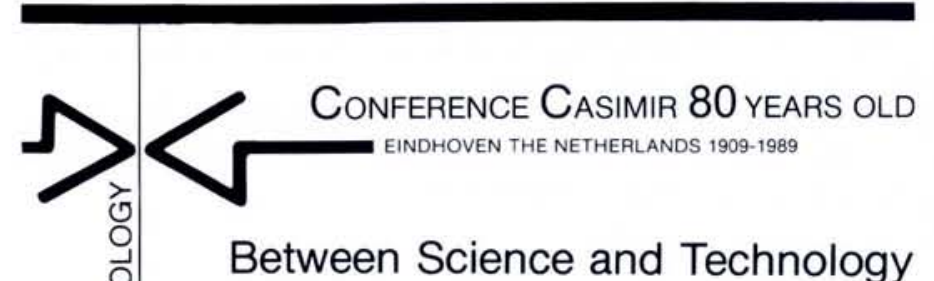

Scientific conference, under the auspices of the Royal Netherlands Academy of Sciences on 29 and 30 June 1989 organised at the Eindhoven University of Technology.

This two-day congress is devoted to the various relationships between fundamental research and technological developments.

Speakers:

- prof.dr. M.F.H. Schuurmans TU Delft

- dr. J.E. Demuth IBM Research Division

prof.dr. G. 't Hooft RU Utrecht

- prof.dr. H.P.J. Haken Universiteit Stuttgart

- prof.dr. L. Hoddeson Universiteit Illinois

dr. J.S. Bell

prof.dr. D. de Wied RU Utrecht

- dr. A. Sarlemijn TU Eindhoven

- profdr. L. van Hove CERN

Place:

Eindhoven University of

Technology

Registration and information:

Congress office EUT

P.O. Box 513

$5600 \mathrm{MB}$ Eindhoven

Conference fee

- Dfl. 200,-
The Netherlands Phone (0) 40-47 4000

\section{METALS \& MATERIALS FOR RESEARCH \& INDUSTRY}

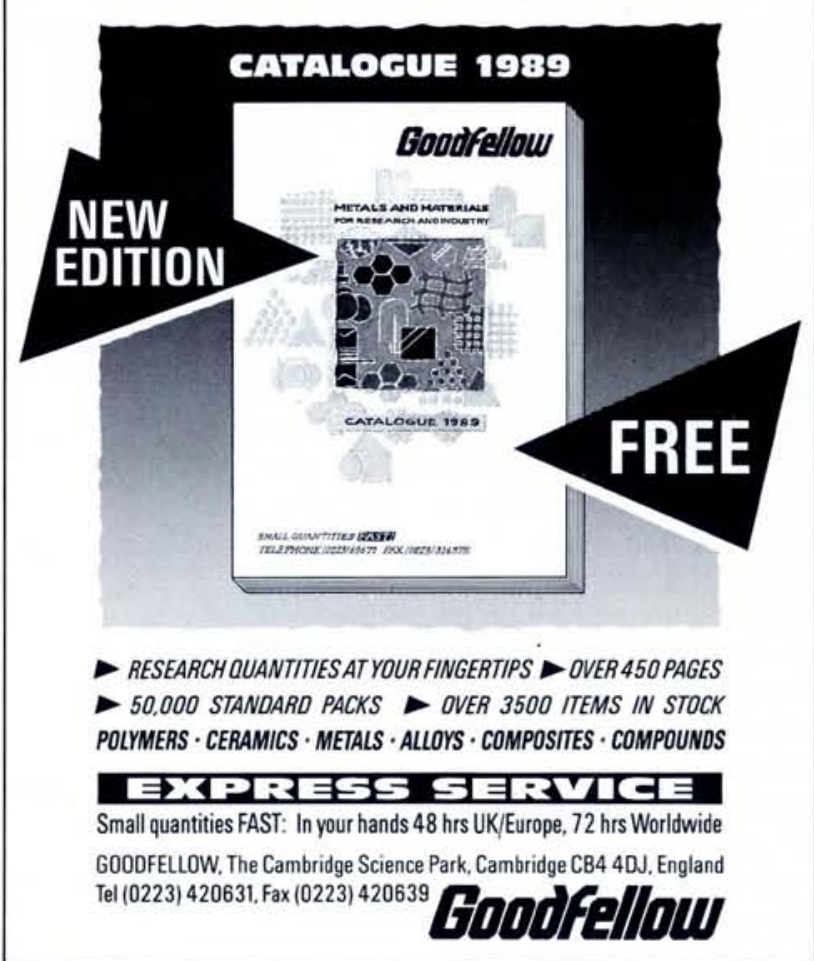


The acceleration of electrons is due to a strongly elevated electrical field near the anode. Probe measurements [8] give spatial potential distributions with high accuracy, see Fig. 3, and make the positive potential near the anode quite obvious. This positive potential is generated by the accumulation of positive charge carriers which enhance the electrical field strength.

There is another way of visualising the accumulation of positive ions near the anode. By local Doppler-shift measurements [8] using laser-induced fluorescence signals, the ion velocity in front of the anode has been determined in the scrape-off mode, see Fig. 6. For the example shown here the ions approach the anode with an axial velocity of more than $10 \mathrm{~km} / \mathrm{s}$. The positively charged cloud turns round the axial ion velocity into a radial outflow with a speed of the same magnitude. Notice, that also an influx of ions is observed at a position less distant to the cathode than that of the positive charge cloud. Thus the particle acceleration is caused actually by an electrical double layer.

\section{Turbulence of the HCA Plasma}

Optical probes and collective forward scattering experiments give the possibility of studying the turbulence of the plasma state [9]. Near the cathode orifice and in front of the anode the turbulence level is high. Typical $\vec{k}$ values of the fluctuations are about $100 \mathrm{~m}^{-1}$, angular frequencies $\omega$ of about $10^{5}-10^{6}$ $\mathrm{rad} / \mathrm{s}$ are common. Near the cathode orifice, so-called universal drift instabilities prevail. These instabilities are driven by the strong radial electron density and temperature gradient occurring there.

\section{HCA, a Diagnostic Test Chamber}

The prolonged steady operation of HCA discharges makes them very interesting for developing and improving plasma diagnostic techniques. Electron density and temperature profiles as shown in Figs. 4 and 5 have been measured by detecting the laser light of a frequency doubled Nd-YAG laser after scattering by free electrons (Thomson scattering). At high laser repetition rates, a photon counting technique allows one to evaluate scattered signals from $10^{9}$ electrons [10] and delivers electron density and temperature with high spatial resolution. The frequency-resolved scattered signals show also the contribution of bound electrons (Rayleigh scattering). Thus the concentration of ground state atomic and ionic species can be measured simultaneously.
A diagnostic based on laser-induced fluorescence [8] is a very versatile technique for measuring the local velocity components, magnetic and electric fields and population densities of selected atomic and ionic states. Molecular properties may be studied advantageously by coherent anti-Stokes Raman scattering (CARS). This method is in vivid development and can be used to study plasma-wall effects at the anode that produce molecular species. CARS as a plasma diagnostic tool delivers local density values, and rotational and vibrational temperatures of distinct species. From this, information about the release of molecular species from solid targets might be gathered.

HCA allows us to produce relatively high fluxes of neutral hydrogen. Thus hydrogen loading of metals and penetration experiments can be performed. These investigations are of importance for fusion oriented devices like tokamaks.

HCA delivers a plasma which starts from a point source and expands then, following the $B$-lines, into a cone. The accurate measurement of charge carrier density distribution means we can determine the anomalous diffusion of electrons across the magnetic field lines.

\section{Summary}

Hollow cathode arcs with superimposed magnetic fields allow us to provide a steady production of plasma with electron temperatures from 1 to $10 \mathrm{eV}$ and charge carrier densities up to $10^{21} \mathrm{~m}^{-3}$ in nearly all working gases. The plasma production within the inaccessible hollow cathode is roughly understood. The actual arc column, the main part of the discharge, is relatively easy to understand if the magnetic field constricts the discharge so that it is confined to the anode diameter. At moderate fields and large inter-electrode spacing, the HCA column diameter exceeds the anode geometry. Then scrape-off effects and enhanced electron energies occur. Amongst the variety of applications opened up the plasma diagnostic potential of HCA is discussed. Associated with highly developed laser light scattering experiments, plasma-wall interaction processes can be studied with high accuracy. Another important application of HCA is as a tool for measuring anomalous diffusion processes induced by plasma turbulence.

\section{REFERENCES}

[1] Theuws P.G.A., Beijerinck H.C.W., Verster N.F. and Schram D.C., J. Phys. E 15 (1982) 573.

[2] Goebel D.M. and Forester A.T., Rev. Sci. Instr. 53 (1982) 810.

[3] Kureichik K.P., J. Appl. Spectrosc. 36 (1982) 627.

[4] Boeschoten F. and Demeter L.J., Plasma Phys. 10 (1968) 391.

[5] Jánossy M., Rózsa K. and Csillag L., Europhys. News 13 (1982) 2, 9.

[6] Delcroix J.L. and Trindade A.R., Advances in Electronics and Electron Physics 35 (1974) 87.

[7] Baksht F.G. and Rybakov A.B., Sov. Phys. Tech. Phys. 28 (8) (1983) 909.

[8] Gräfen W., v. Hoesslin M., Kempkens H. and Uhlenbusch J., Phys. of Fluids, in print. [9] Pots B.F.M., Thesis Eindhoven Univ. (1979).

[10] Jauernik P., Kempkens H. and Uhlenbusch J., Plasma Phys. Controlled Fusion 29 (1987) 1615.

Fig. 6 - Spatial distribution of the ion velocity components $V_{r}$ and $v_{r}+v_{z}$ in front of the anode for the long arc mode after [10], denotation and parameters see Fig. 3.

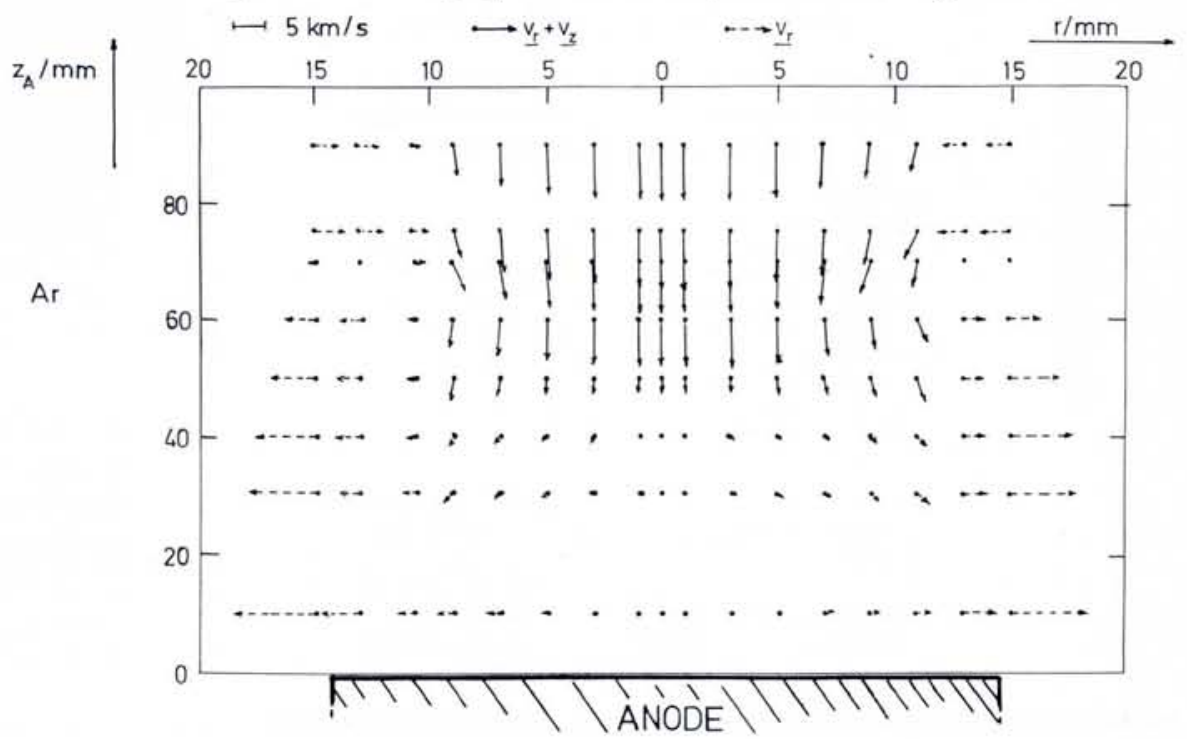

